\title{
Boosting the Performance of Solar Cells with Intermediate Band Absorbers-The Case of ZnTe:O
}

\author{
Othmane Skhouni ${ }^{1}$, Ahmed El Manouni ${ }^{1}$, Hamza Bayad ${ }^{1}$ and Bernabe Mari ${ }^{2}$ \\ 1. Laboratory of Atmospheric Physics, Materials and Modeling (LPAMM), Department of Physics, University Hassan II, Faculty of
} Sciences and Technics, Mohammedia 20650, Morocco

2. Department of Applied Physics-ETSED, Polytechnic University, Valencia, Camí de Vera s/n 46022, Spain

Received: April 13, 2017 / Accepted: April 20, 2017 / Published: June 30, 2017.

\begin{abstract}
This work reports on modeling IB (intermediate band) solar cells based on ZnTe:O semiconductor and determination of their photovoltaic parameters using SCAPS (solar cell capacitance simulator) software. A comparative study between photovoltaic performance of $\mathrm{ZnTe}$ and $\mathrm{ZnTe}: \mathrm{O}$ based solar cells has been carried out. It has been found that the energy conversion efficiency $\eta$, short-circuit current density $J_{\mathrm{sc}}$, EQE (external quantum efficiency) and FF (fill factor) increased with increasing oxygen doping concentration $N_{t}$ up to the shallow acceptor density $N_{A}$ and decreased when $\mathrm{N}_{\mathrm{t}}$ was higher than $N_{A}$. The open circuit-voltage $V_{o c}$ remained constant for $N_{t}$ lower than the acceptor doping concentration $N_{A}$ and decreased for $N_{t}$ higher than $N_{A}$. The increase of $\eta, J_{s c}$ and $F F$ is due to the fact that IB is fully empted, so sub-bandgap photons can be absorbed by hole photoemission process from the VB (valence band) to the IB. The decrease of $\eta, J_{\mathrm{sc}}, \mathrm{EQE}$ and $F F$ is attributed to overcompensation for the base doping $N_{A}$ making electron photoemission process from IB to the $\mathrm{CB}$ (conduction band) maximized. This indicates that there is a competition between oxygen doping and intrinsic acceptor defects. The optimal concentrations of oxygen and shallow acceptor carriers were found to be $N_{t} \approx 10^{15} \mathrm{~cm}^{-3}$ and $N_{A} \approx 10^{14} \mathrm{~cm}^{-3}$. The corresponding photovoltaic parameters were $\eta=41.5 \%, J_{\mathrm{sc}}=31.2 \mathrm{~mA} / \mathrm{cm}^{2}, V_{\mathrm{oc}}=1.80 \mathrm{~V}$ and $F F=75.1 \%$. Finally, the EQE spectra showed a blue shift of absorption edge indicating that the absorption process is extended to the sub-bandgap photons through IB.
\end{abstract}

Key words: ZnTe:O, IB solar cell, energy conversion efficiency, current-voltage characteristic, SCAPS.

\section{Introduction}

Single-junction photovoltaic solar cells are the most commonly used devices in solar industry, but its theoretical maximum efficiency is about $32 \%$ under an unconcentrated sunlight or $41 \%$ under concentrated sunlight [1]. This is due to that semiconductors absorb only photons of energy greater than their bandgap energy, loss of the excess energy by thermalization process and radiative and non radiative recombination processes. Then efforts have been done to increase the conversion efficiency by realizing multijunction solar cells, but without success since the photovoltaic efficiency remained in order of magnitude that is

Corresponding author: Ahmed El Manouni, professor, research fields: materials and thin films for solar energy. based on semiconductor PN junction solar cells. Luque and Martí [2, 3] proposed another high-efficiency concept: IBSC (intermediate band solar cell). It consists of $p$-IB-n structure with the p-type and n-type regions formed from a large-bandgap semiconductor (bandgap ideally $1.9 \mathrm{eV}$ ) and the IB (intermediate band) region consisting of a modified semiconductor with a large bandgap and an extra set of allowed electronic levels entirely contained inside the bandgap. The extra electronic states form an IB which allows the generation of one net electron-hole pair when sub-bandgap photons are absorbed: VB (valence band) to IB and IB band to CB (conduction band) transitions, additionally to the band-band optical transition. A necessary requirement is that IB is half-filled with electrons to provide both 
empty state to receive electrons from VB and filled states to provide electrons to be supplied to CB [4-7]. These three photon absorption processes allow to cover the entire solar spectrum and to increase the photovoltaic efficiency of IBSC compared to the based semiconductor PN junction solar cells with a single optical transition. The theoretical photovoltaic efficiency limit for IB solar cells is predicted to be $63.2 \%$ for the $6,000 \mathrm{~K}$ black body spectrum [2, 5] or 49.4\% under AM 1.5 G illumination [3].

Recently, several engineered semiconductor systems have been widely studied and it reported to display the IB band structure. The most is the class of highly-mismatched alloys, including quantum dots $[8$, 9], dilute nitrides [10, 11] and impurity doping semiconductors $[5,12]$. Zinc telluride compound $\mathrm{ZnTe}$ is one of semiconductors which attract most attention for low-cost photovoltaic conversion [13, 14], however, solar cells based on this material have produced a limited theoretical photovoltaic conversion efficiency about $10 \%[15,16]$ due to its high bandgap and zinc vacancy native defects in ZnTe material. In order to increase the conversion efficiency new semiconductor materials with sub-band optical absorption have been proposed [5, 12]. The incorporation of oxygen atoms into $\mathrm{ZnTe}$ semiconductor leads to the formation of electronic states located within the bandgap at approximately 1.8-1.9 eV above the $\mathrm{VB}$ edge as a result of substitution of $\mathrm{O}$ in Te sites in the ZnTe lattice [5, 17]. These extra electronic states form an IB which divides the bandgap into two sub-band allowing therefore two new optical transitions: from VB to IB and from IB to $\mathrm{CB}$, beside the band-band transition as shown in Fig. 1. It results in a broad response to the solar spectrum, a reduction of energy losses due to thermal relaxation of photogenerated carriers, highly radiative properties and long carrier lifetimes [18].

In this paper, ZnTe:O IB solar cell has been modeled and its photovoltaic performance has been calculated using SCAPS (solar cell capacitance

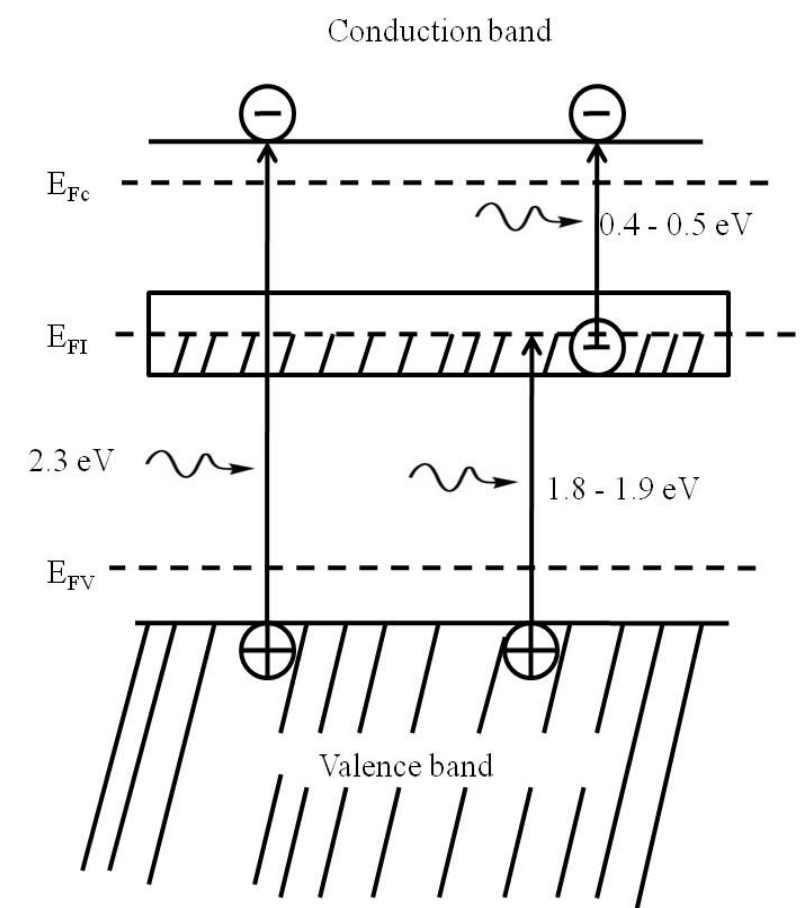

Fig. 1 Schematic of optical transitions for ZnTe:O intermediate band solar cell.

simulator) software. The effect of oxygen doping on mean photovoltaic characteristics has been studied and the optimal oxygen concentration has be determinate. Finally, a comparative study of photovoltaic parameters of ZnTe and IB-ZnTe:O thin films solar cells has been performed to demonstrate the oxygen doping interest to achieve ZnTe:O IB semiconductor with attractive photovoltaic properties including high conversion efficiency.

\section{Basic Equations}

The numerical SCAPS software is a one dimensional solar cell simulation program. It was designed as a general polycrystalline thin-film device simulator, and has been used for modeling solar cells based on conventional thin film semiconductors such as CIS, CIGS and CdTe [19]. It can also be used to set a composition grading of various material parameters in a solar cell. More recently it allows simulating IBSC taking into account the absorption of sub-band photons, IPSC (impurity photovoltaic solar cells) and perovskyte based solar cells. 
On the other hand, SCAPS software with appropriates boundary conditions allows mainly to:

- Estimate the steady-state energy band diagram, recombination profile, carrier transport in $1 \mathrm{D}$;

- Calculate the recombination currents using the SRH (Shockley-Read-Hall) model for bulk and interface defects;

- Determinate spectral response, $J_{\mathrm{SC}}-V, C-V, C-f$ and $Q-V$ characteristics. Numerical calculations can be done under light and/or dark conditions and as a function of temperature.

In order to simulate output photovoltaic parameters, the proposed solar cell is divided into $\mathrm{N}$ intervals. In each interval, SCAPS resolves the well known basic semiconductor equations in a perpendicular direction to the cell: the Poisson's equation and the continuity equations for electrons and holes, using finite-difference method.

The Poisson's equation is

$$
\frac{\partial^{2} \psi(x)}{\partial x^{2}}=\frac{e}{\varepsilon_{r} \varepsilon_{0}}\left[p(x)-n(x)+N_{D}^{+}-N_{A}^{-}+p_{t}-n_{t}\right]
$$

where, $\psi$ is the electrostatic potential, $e$ is the electrical charge, $\varepsilon_{r}$ is the relative permittivity, $\varepsilon_{0}$ is the vacuum permittivity, $p$ and $n$ are hole and electron concentrations, $N_{\mathrm{D}}$ and $N_{\mathrm{A}}$ are the donor and acceptor concentrations, $p_{t}$ and $n_{t}$ are trapped hole and electron concentrations, respectively.

The continuity equations for electrons and holes are

$$
\begin{gathered}
\frac{d J_{n}(x)}{d x}-e \frac{\partial n(x)}{\partial t}-e \frac{\partial n_{t}}{\partial t}=G_{n}(x)-R_{n}(x) \\
\frac{d J_{p}(x)}{d x}-e \frac{\partial p(x)}{\partial t}-e \frac{\partial p_{t}}{\partial t}=G_{p}(x)-R_{p}(x)
\end{gathered}
$$

where, $J_{n}$ and $J_{p}$ are electron and hole current densities, $G_{i}(x)$ and $R_{i}(x)$ are charge generation and recombination rates ( $i=n$ for electrons and $i=p$ for holes).

SCAP calculates solutions of Poison's equation for electrostatic potential $\psi$ and the continuity equations for electrons and holes at steady state with boundary conditions $[20,21]$ and in the frequency domain [22, 23].

\section{Design of ZnTe:O Based Solar Cell}

The schematic of the IB-ZnTe:O solar cells used in the simulation is shown in Fig. 2. Its structure is $\mathrm{ZnO}$ (n-type)/CdS(n-type)/IB-ZnTe:O

(p-type)/ZnTe $\left(\mathrm{p}^{+}\right.$-type)/metal. The IB ZnTe:O thin film is inserted between $\mathrm{P}^{+}-\mathrm{ZnTe}$ and $\mathrm{n}-\mathrm{CdS}$ layers and used as an absorber layer with thickness about $0.7 \mu \mathrm{m}$ and oxygen concentration variable from $10^{14}$ to $10^{19} \mathrm{~cm}^{-3}$. The $\mathrm{n}$-CdS layer is used as a front contact for IB-ZnTe:O absorber and as a buffer for the solar cell device with thickness of $0.08 \mu \mathrm{m}$ and donor carrier density of $N_{D}=$ $10^{18} \mathrm{~cm}^{-3}$. The $\mathrm{P}^{+}$-ZnTe material is inserted at the back surface of the absorber and it has been added to produce a BSF (back surface field) to inhibit the minority carrier loss at the back contact of the cell. The acceptor carrier density of $\mathrm{P}^{+}-\mathrm{ZnTe}$ film is $10^{19}$ $\mathrm{cm}^{-3}$ and its thickness is $0.1 \mu \mathrm{m}$. The $\mathrm{n}-\mathrm{ZnO}$ layer is used as a window layer with thickness of $0.05 \mu \mathrm{m}$ and donor carrier density of $N_{D}=10^{18} \mathrm{~cm}^{-3}$. The front and back contacts of the modeled IB-ZnTe:O thin film solar cell are in aluminum and platinum, respectively. The design is presented in the substrate configuration and the illumined side of the modeled solar cell is the $\mathrm{ZnO}$ window.

\section{Input Parameters}

\subsection{Layer and Defect Parameters}

In order to simulate photovoltaic performance of the modeled $\mathrm{ZnO} / \mathrm{CdS} / \mathrm{ZnTeO} / \mathrm{ZnTe} /$ metal solar cell, several parameters relative to all layers of the cell have been introduced. The input parameters of $\mathrm{ZnO}$ and CdS layers and of front and back contacts are the same as those used in our previous work [16]. Additional input parameters relative to IB-ZnTe:O absorber, $\mathrm{P}^{+}$-ZnTe layer and oxygen defect have been introduced [18, 24-26]. They are shown in Table 1. The oxygen concentration $N_{t}$ was ranging from $10^{14}$ to $10^{19} \mathrm{~cm}^{-3}$ and thermal and optical capture cross 


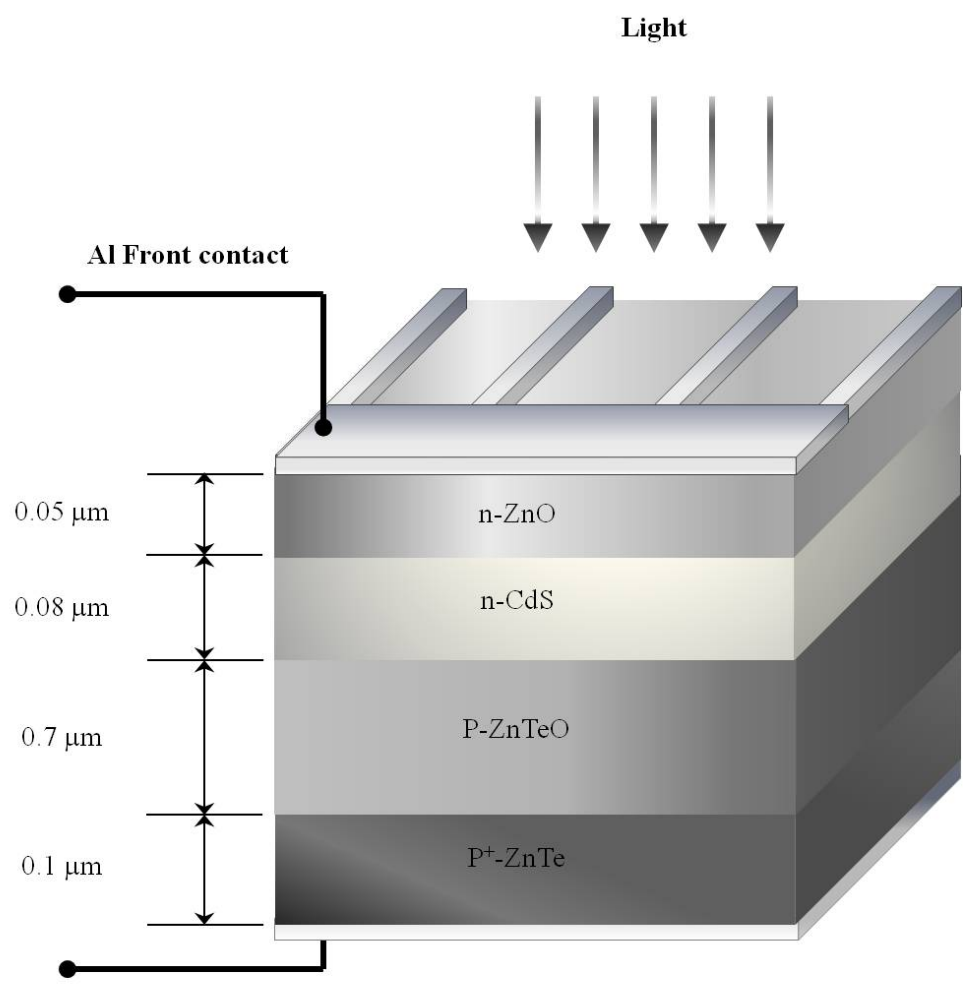

Pt Back contact

Fig. 2 Solar cell device structure based on IB-ZnTe:O thin films.

Table 1 Input parameters used in simulation of $\mathrm{ZnO} / \mathrm{CdS} / \mathrm{ZnTe}: \mathrm{O} / \mathrm{ZnTe}$ solar cells.

\begin{tabular}{lll}
\hline P-ZnTe:O layer properties & Symbol & Value \\
\hline Thickness & $x(\mu \mathrm{m})$ & 0.7 \\
Band gap energy & $E g(\mathrm{eV})$ & 2.30 \\
Electronic affinity & $X_{e}(\mathrm{eV})$ & 3.73 \\
Dielectric constant & $\varepsilon$ & 10.3 \\
CB effective density of state for electron & $N c\left(\mathrm{~cm}^{-3}\right)$ & $1.176 \times 10^{18}$ \\
VB effective density of states & $N v\left(\mathrm{~cm}^{-3}\right)$ & $1.166 \times 10^{19}$ \\
Electron thermal velocity & $v_{n}^{t h}(\mathrm{~cm} / \mathrm{s})$ & $3.24 \times 10^{7}$ \\
Hole thermal velocity & $v_{p}^{t h}(\mathrm{~cm} / \mathrm{s})$ & $1.51 \times 10^{7}$ \\
Electron mobility at 300 K & $\mu_{n}\left(\mathrm{~cm}^{2} / \mathrm{v} \cdot \mathrm{s}\right)$ & 330 \\
Hole mobility at 300 K & $\mu_{h}\left(\mathrm{~cm}^{2} / \mathrm{v} \cdot \mathrm{s}\right)$ & 80 \\
Shallow uniform donor density & $N_{D}\left(\mathrm{~cm}^{-3}\right)$ & 0 \\
Shallow uniform acceptor density & $N_{A}\left(\mathrm{~cm}^{-3}\right)$ & $10^{13}-10^{16}$ \\
Impurity oxygen properties & & 1.8 \\
Oxygen level energy respect to E & $E_{\mathrm{v}}-E_{V}(\mathrm{eV})$ & $10^{14}-10^{19}$ \\
Oxygen concentration & $N_{t}\left(\mathrm{~cm}^{-3}\right)$ & $10^{-15}$ \\
Thermal capture cross section of electron & $\sigma_{n}^{t h}\left(\mathrm{~cm}^{2}\right)$ & $10^{-15}$ \\
Thermal capture cross section of hole & $\sigma_{p}^{t h}\left(\mathrm{~cm}^{2}\right)$ & $\sigma_{n}^{o p t}\left(\mathrm{~cm}^{2}\right)$ \\
Optical capture cross section of electron & $\sigma_{p}^{o p t}\left(\mathrm{~cm}^{2}\right)$ & Calculated from \\
Optical capture cross section of hole & model [24] \\
\hline
\end{tabular}


sections of electrons and holes have been calculated using the Lucovsky model [24]. Furthermore, the IB was considered as a located and isolated band to neglect the effect of the charge transport along this band and as a discrete level for assimilating it to an impurity photovoltaic as suggested by Tobias et al. [25].

\subsection{Absorption Coefficient}

To calculate photovoltaic performance of the modeled IB-ZnTe:O thin film based solar cell by SCAPS program a new absorption coefficient has been simulated taking in account sub-bandgap absorption process. In the calculations, a theoretical model based on double Boltzmann sigmoid functions was used to fit the absorption coefficient of IB-ZnTe:O semiconductor. Fig. 3 shows the obtained spectrum; the absorption coefficient curve of $\mathrm{ZnTe}$ semiconductor is shown for comparison. One can observe that the absorption spectrum of ZnTe:O shows an absorption edge at $2.3 \mathrm{eV}$ corresponding to the band-band absorption of $\mathrm{ZnTe}: \mathrm{O}$ semiconductor and an additional absorption edge at $1.8 \mathrm{eV}$ corresponding to optical transition from VB to IB. The absorption edge at $0.5 \mathrm{eV}$ corresponding to IB-CB transition is very weak and not seen here.

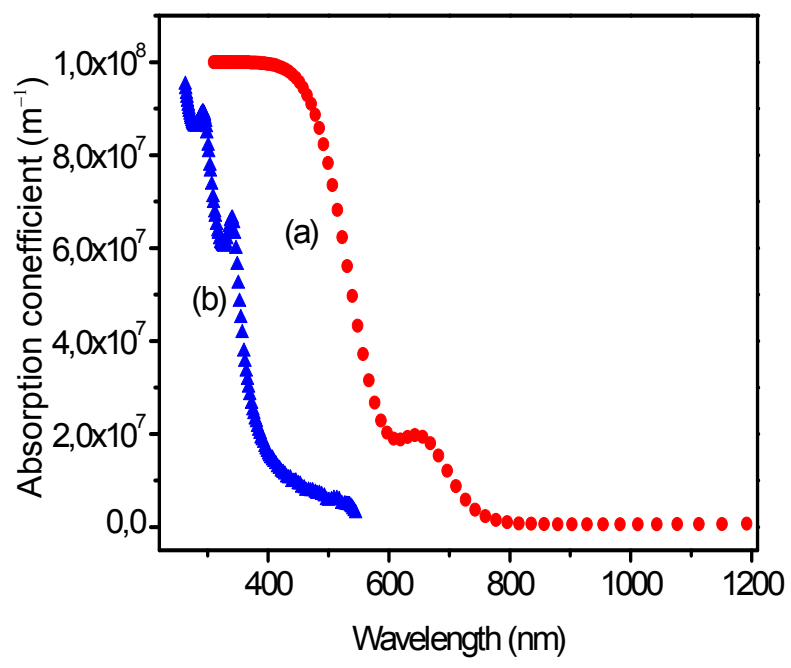

Fig. 3 Absorption coefficient spectra.
(a) ZnTe:O (simulated)
(b) ZnTe (extracted from SCAPS)

\section{Results and Discussion}

The main objective of this work is modeling solar devices based on an IB ZnTe:O thin film and then determination of the effect of IB on photovoltaic performance. The optimal oxygen concentration photovoltaic characteristics are also investigated. The calculations are done for IB-ZnTe:O thickness of about $0.7 \mu \mathrm{m}$ and at temperature of $300 \mathrm{~K}$.

\subsection{Energy Band Diagram}

SCAPS software allows simulating the energy band diagram of IBSC when the absorption coefficient is known. Fig. 4 shows the energy band diagram of the modeled $\mathrm{ZnO} / \mathrm{CdS} / \mathrm{IB}-\mathrm{ZnTe}: \mathrm{O} / \mathrm{ZnTe}$ solar cell generated by SCAPS program at the dark and zero bias voltage. The energy levels are shown with respect to the Fermi level, which is set to $0 \mathrm{eV}$ in the band diagram.

The $\mathrm{n}$ type, IB and p-type regions and the associated $\mathrm{VB}$ and $\mathrm{CB}$ edges and the interfaces and their positions are clearly shown in Fig. 4. It is observed that an abrupt junction is formed at the $\mathrm{CdS} / \mathrm{ZnTe}: \mathrm{O}$ interface. Also, there is no potential barrier between $\mathrm{CdS}$ and $\mathrm{ZnTe}: \mathrm{O}$ layers for electrons and holes drifting from $\mathrm{p}$-side to $\mathrm{n}$-side and from $\mathrm{n}$-side to $\mathrm{p}$-side in the junction, respectively. This should improve the collected current and the open voltage $V_{\text {oc }}$. A Schottky barrier with height about $0.195 \mathrm{eV}$ appears at $\mathrm{ZnTe} \mathrm{O} / \mathrm{ZnTe}$ interface. This barrier allows to reduce carrier loss at the back contact thanks to a BSF produced by $\mathrm{P}^{+}-\mathrm{ZnTe}$ layer inserted at the back surface of the ZnTe:O absorber. The BSF effect should contribute to improving photovoltaic performance of the simulated IB-ZnTe:O solar cells. It is also seen that IB is set at $1.8 \mathrm{eV}$ above the VB edge.

\section{$5.2 \mathrm{~J}$-V Characteristics}

The variation of current as function of voltage for $\mathrm{ZnTe}$ and IB-ZnTe:O based solar cells at the dark and under AM1.5 standard illumination is shown in Fig. 5. The curves are plotted for different oxygen 


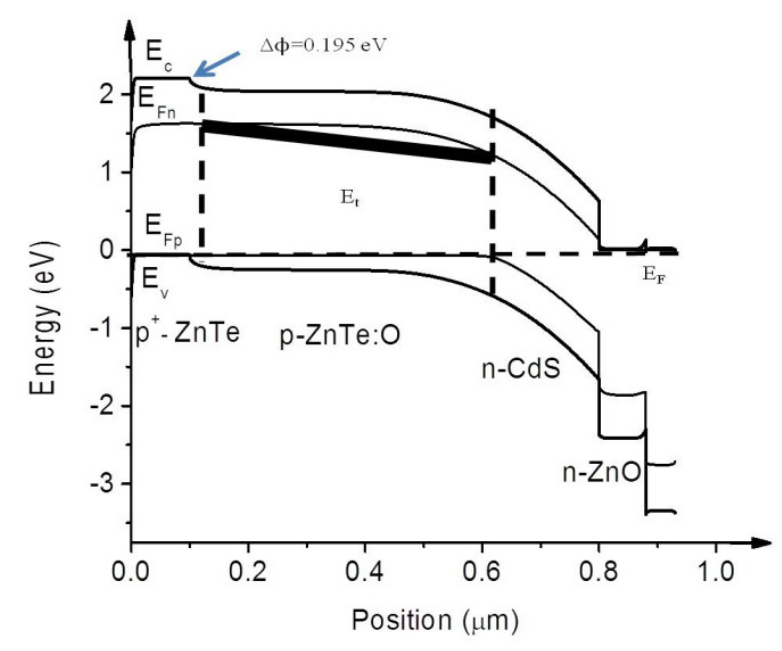

Fig. 4 Schematic band diagram of ZnTe:O intermediate band solar cell generated by SCAPS software at the dark and zero bias voltage.

concentrations ranging from $10^{14}$ to $10^{19} \mathrm{~cm}^{-3}$ using parameters relative to different layers given in Table 1. The internal back and front reflexion coefficients were set to $R_{f}=R_{b}=0.98$. All curves show a cross-over effect and an increasing of the current as the voltage increases indicating that the current depends on voltage and carrier transport mechanism. The J-V characteristic in the dark is typical of a Schottky diode with a current minimum due to the minority carriers. Under AM1.5 irradiation, a clear photovoltaic response is observed for both $\mathrm{ZnTe}$ and IB-ZnT:O thin film devices with raised current for the
IB-ZnTe:O based solar cell. This high current of $\mathrm{ZnTe}: \mathrm{O}$ based solar cell is due to the large photogenerated carriers and extend absorption to the sub-gap photons. Also, it is observed that as the current density increases with increasing oxygen concentration from $N_{t}=0 \mathrm{~cm}^{-3}$ (for ZnTe based device) to $N_{t}=10^{15} \mathrm{~cm}^{-3}$ and then it decreases for oxygen concentration varying from $10^{16}$ to $10^{19} \mathrm{~cm}^{-3}$. At oxygen concentration $N_{t}=10^{19} \mathrm{~cm}^{-3}$ the current becomes lower than that generated by $\mathrm{ZnTe}$ solar cell. From this analysis, it can be considered that the optimal oxygen concentration is $10^{15} \mathrm{~cm}^{-3}$ which gives an IB effect with a maximum current density.

On the other hand, it is known that high series resistance and low shunt resistance of the solar cells degrade photovoltaic performance. So realizing a solar cell with lower series resistance and high shunt resistance provides to produce high energy conversion efficiency. In this sense, the IB-ZnTe based solar cells were modeled by an equivalent electric circuit to determinate the shunt resistance and series resistance values. In our case, the calculated values depend on both shallow acceptor density $N_{A}$ and oxygen concentration $N_{t}$ and the $R_{S}$ and $R_{s h}$ were determined to be $12.2 \Omega \cdot \mathrm{cm}^{2}$ and $33,63,22 \mathrm{~K} \Omega \cdot \mathrm{cm}^{2}$ for $N_{A}=10^{14}$ and $N_{t}=10^{15} \mathrm{~cm}^{-3}$, respectively.

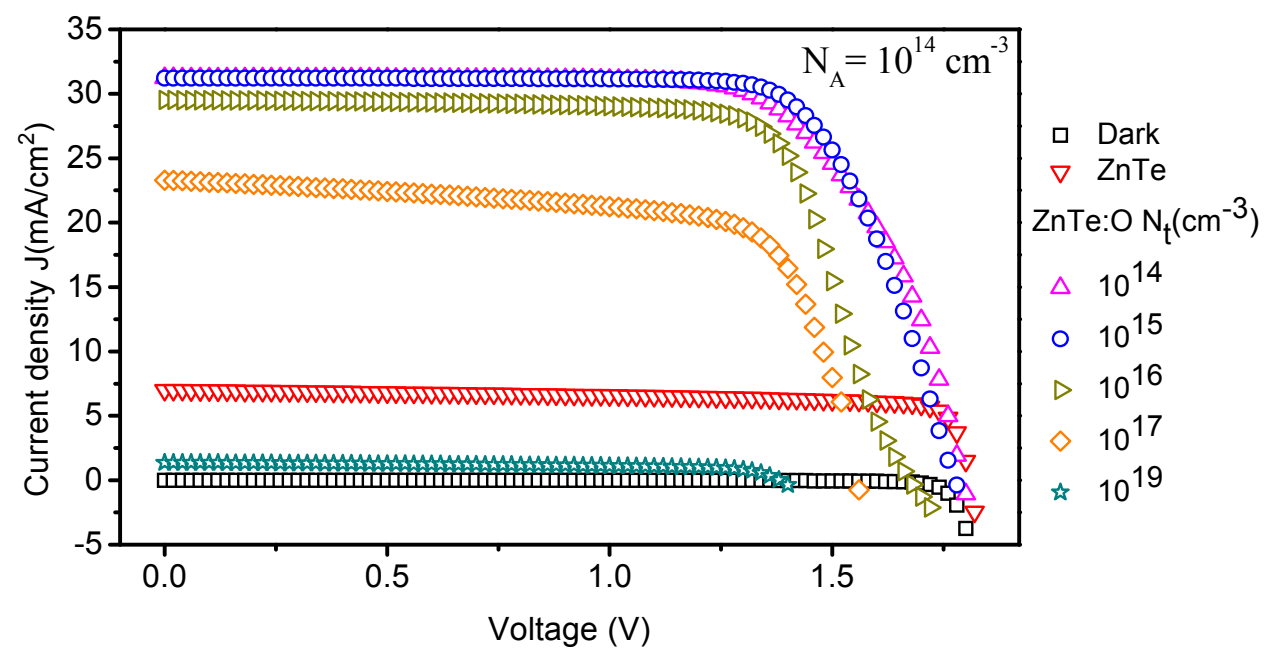

Fig. 5 Current-voltage characteristics for ZnTe and IB-ZnTe:O based solar cells in the dark and under AM 1.5 illumination for different oxygen concentrations and $\left(E_{t}-E_{v}\right)=1.8 \mathrm{eV}$. 

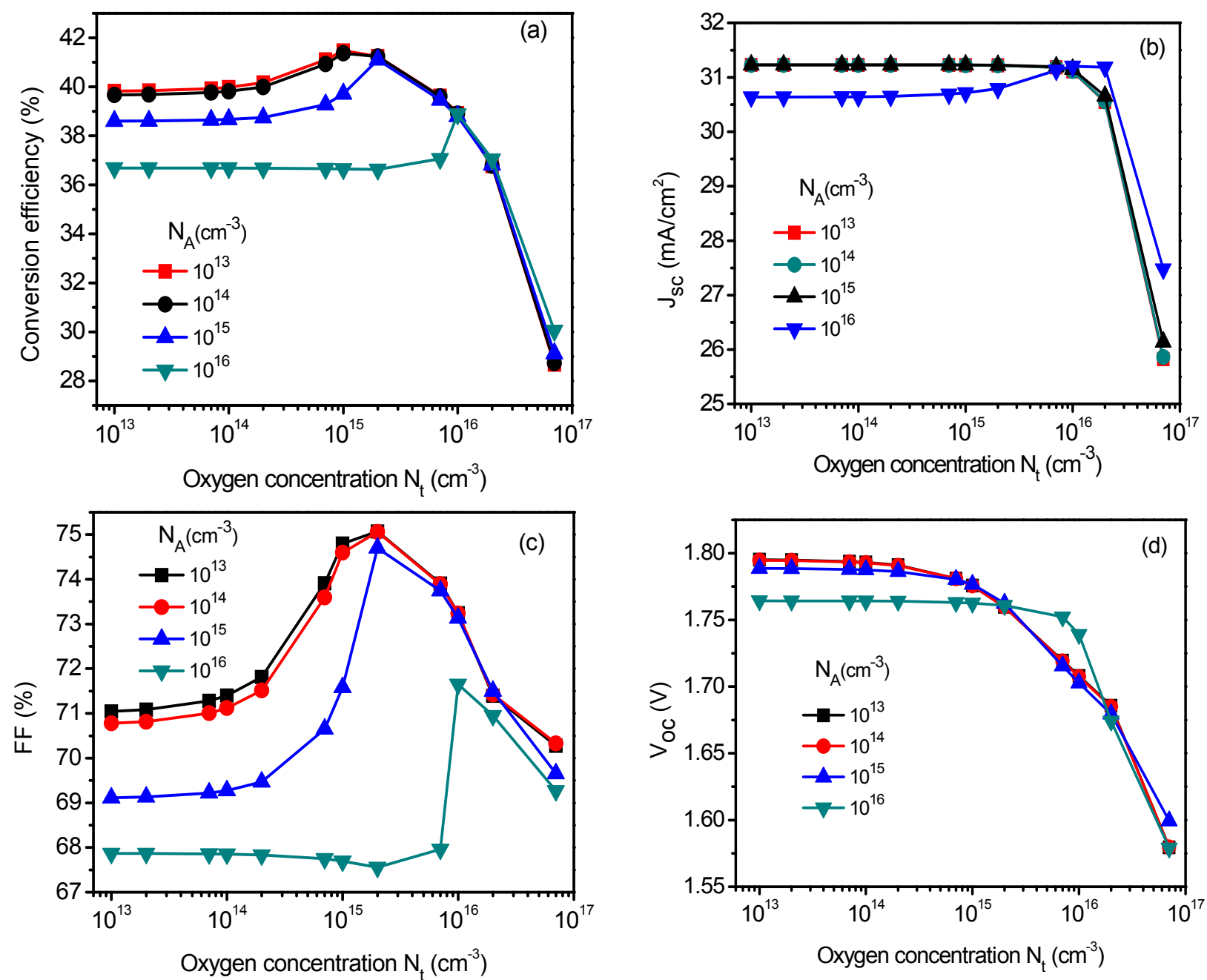

Fig. 6 Dependence of a) energy conversion efficiency $\eta$, b) short circuit current density $J_{s c}$, c) fill factor $F F$, d) open circuit voltage $V_{\text {oc }}$ on oxygen density in ZnTe:O for IB located at $1.8 \mathrm{eV}$ above the $\mathrm{VB}$ edge and for different base doping $N_{A}$.

\subsection{Oxygen Density Effect}

The physical processes associated with the IB will depend on the nature and density of the IB electronic states and will be material and process specific. In this respect, we have studied the effect of oxygen density of states in IB on the photovoltaic output parameters of $\mathrm{ZnO} / \mathrm{CdS} / \mathrm{ZnTe}: \mathrm{O} / \mathrm{ZnTe}$ solar cells. Fig. 6 shows the variation of short-circuit current density $J_{s c}$, open circuit voltage $V_{o c}$, fill factor $F F$ and energy conversion efficiency $\eta$ as function of oxygen concentration $N_{t}$ for IB energy achieving $\left(E_{t}-E_{v}\right)=1.8 \mathrm{eV}$ and for different shallow acceptor density $N_{A}$. From this figure, it is observed that for each base doping $N_{A}$ the $\eta, F F$ and $J_{s c}$ increase until a maximum with increasing the oxygen concentration up to $N_{t} \approx N_{A}$ and then decrease for oxygen concentration $N_{t}$ higher than the shallow acceptor density $N_{A}\left(N_{t}>N_{A}\right)$. The increase of $\eta, F F$ and $J_{s c}$ is due to the fact that IB is fully empted, so sub-bandgap photons can be absorbed by hole photoemission process from VB to IB. The decrease of $\eta, F F$ and $J_{s c}$ when $N_{t}>N_{A}$ can be explained by an overcompensation for the base doping $N_{A}$ which occurs making electron photoemission process from IB to CB maximized and/or to the reduction both in minority carrier lifetime and diffusion length. The optimal values of $\eta, F F$ and $J_{s c}$ depend on the acceptor density $N_{A}$ and the highest values are obtained for $N_{A}$ $=10^{13} \mathrm{~cm}^{-3}$. Concerning the open circuit voltage $V_{o c}$, it remains constant when oxygen density $N_{t}$ is varied up 
to the basic doping concentration $\left(N_{t} \approx N_{A}\right)$ and then it decreases clearly for $N_{t}$ higher than $N_{A}$. This decreasing of $V_{o c}$ for larger values of $N_{t}$ can be attributed to the fact that when the oxygen concentration exceeds the shallow doping concentration, the semiconductor is compensated and becomes $\mathrm{N}$ type. From this analysis one can conclude that there is a competition between oxygen doping and shallow acceptor concentration to produce an IB effect and the optimal oxygen and acceptor concentrations are $N_{t}=10^{15} \mathrm{~cm}^{-3}$ and $N_{A}=10^{14} \mathrm{~cm}^{-3}$, respectively. The corresponding values of $\eta, J_{s c}$ and $F F$ are $41.47 \%$, $31.20 \mathrm{~mA} / \mathrm{cm}^{2}$ and $75.06 \%$, respectively.

The optimal output parameters of $\mathrm{ZnTe}$ and IB-ZnTe:O thin film based solar cells are presented in Table 2. They were obtained for lower shallow acceptor density of $10^{14} \mathrm{~cm}^{-3}$ and oxygen concentration of $10^{15} \mathrm{~cm}^{-3}$. One can observe that the energy conversion efficiency and the current density have been enhanced by a factor more than 4 as result of an IB effect due to the oxygen doping of $\mathrm{ZnTe}$ semiconductor.

\subsection{External Quantum Efficiency}

A more obvious means of detecting the IB response is through the external quantum efficiency EQE. EQE curves of IB ZnTe:O based solar cells for different doping oxygen $N_{t}$ with shallow acceptor concentration of $N_{A}=10^{14} \mathrm{~cm}^{-3}$ are shown in Fig. 7; the spectrum relative to $\mathrm{ZnTe}$ based solar cell is plotted for comparison. A sharp band edge response is observed at energy of $2.26 \mathrm{eV}$ for ZnTe device with a gradual response decrease with increasing energy. The spectral response curves for IB-ZnTe:O solar cells exhibit an extended response at energies below the bandgap energy of ZnTe material and the response is dominated by sub-bandgap absorption processes: two front absorption edges are observed at energies around 1.8 and $2.3 \mathrm{eV}$ corresponding to $\mathrm{VB}-\mathrm{IB}$ and $\mathrm{VB}-\mathrm{CB}$ optical transitions, respectively. The feature relative to IB-CB optical transition is so weak that it is not seen on the EQE curves. Furthermore, the EQE increases with increasing oxygen concentration $N_{t}$ up to $10^{15}$ $\mathrm{cm}^{-3}$, reaches a maximum at $N_{t}=10^{15} \mathrm{~cm}^{-3}$ and decreases when $N_{t}$ is higher than this value. Again, this indicates that the optimal oxygen doping concentration is $10^{15} \mathrm{~cm}^{-3}$ as already mentioned in the Section 5.3. For oxygen concentration around $10^{19}$ $\mathrm{cm}^{-3}$ the EQE curves show a clear degradation due to an overcompensation of the oxygen impurity for the

Table 2 Optimal output parameters for $\mathrm{ZnTe}$ and IB-ZnTe:O based solar cells.

\begin{tabular}{lllll}
\hline Photovoltaic characteristics & $\eta(\%)$ & $J_{\text {sc }}\left(\mathrm{mA} / \mathrm{cm}^{2}\right)$ & $F F(\%)$ & $V_{\text {oc }}($ Volt $)$ \\
\hline ZnTe & 10 & 7.01 & 78.84 & 1.81 \\
ZnTe:O & 41.47 & 31.20 & 75.06 & 1.795 \\
\hline
\end{tabular}

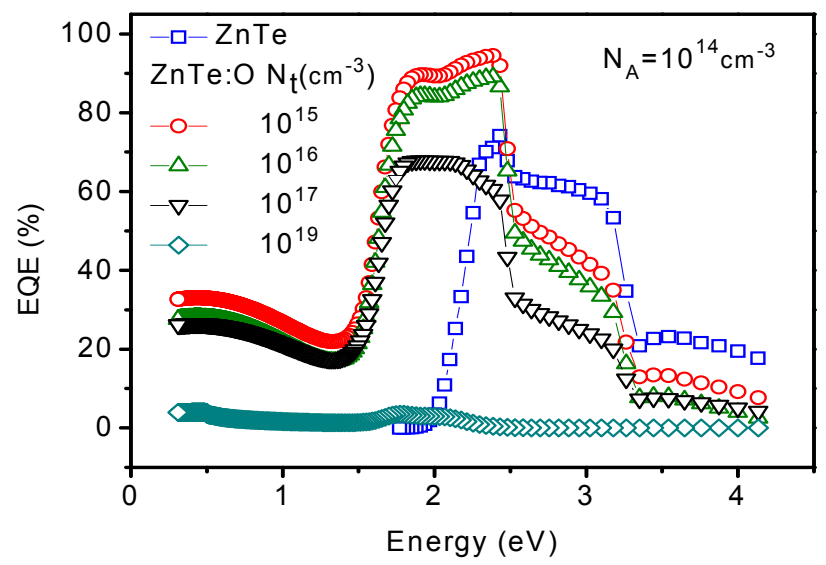

Fig. 7 Response spectral of $\mathrm{ZnTe}: O$ and $\mathrm{ZnTe}$ based solar cells for different oxygen impurity concentrations and $\left(E_{t}-E_{v}\right)=$ $1.8 \mathrm{eV}$. 
base doping making electron photoemission from IB to the $\mathrm{CB}$ dominate and/or to the reduction both in minority carrier lifetime and diffusion length. Consequently, to produce IB effect oxygen doming concentration must be controlled and an optimal concentration must be introduced.

\section{Conclusions}

The introduction of $\mathrm{O}_{\mathrm{Te}}$ substitutional impurities in ZnTe semiconductor provides a path for sub-bandgap optical absorption without a significant sacrifice in the optical and electronic properties of the material. These transitions may be effectively utilized for an IB solar cell material.

IB-ZnTe:O solar cells have been modeled and its photovoltaic performance has been calculated using SCAPS 1D-program. A comparative analysis between $\mathrm{ZnTe}$ and ZnTe:O based solar cells has been performed. It results in that the oxygen doping of $\mathrm{ZnTe}$ absorber has induced an enhancement of current and energy conversion efficiency by a factor more than 4 and the oxygen doping amount must be controlled because the results have shown a competition between oxygen and shallow acceptor densities to produce an IB effect. The optimal oxygen and acceptor carrier densities were found to be $10^{15}$ and $10^{14} \mathrm{~cm}^{-3}$, respectively. The corresponding photovoltaic parameters are $\eta=41.5 \%, J_{s c}=31.2$ $\mathrm{mA} / \mathrm{cm}^{2}, V_{\text {oc }}=1.80 \mathrm{~V}$ and $F F=75.1 \%$. Finally, an extended spectral response to the sub-bandgap photons through the intermediate has been observed for ZnTe:O based solar cells.

\section{References}

[1] Shockley, W., and Queisser, H. J. 1961. "Detailed Balance Limit of Efficiency of P-N Junction Solar Cells." Journal of Applied Physics 32 (3): 510-9.

[2] Luque, A. and Marti, A. 1997. "Increasing the Efficiency of Ideal Solar Cells by Photon Induced Transitions at Intermediate Levels." Physical Review Letters 78 (June): 5014-7.

[3] Luque, A., and Marti, A. 2001. "A Metallic Intermediate Band High Efficiency Solar Cell." Progress in
Photovoltaics 9 (March): 73-86.

[4] Luque, A., Marti, A., Antolin, E., and Linares, P. G. 2010. "Intraband Absorption for Normal Illumination in Quantum Dot Intermediate Band Solar Cells." Solar Energy Materials and Solar Cells 94 (12): 2032-5.

[5] Wang, W., Lin, A. S., and Phillips, J. D. 2009. "Intermediate-Band Photovoltaic Solar Cell Based on ZnTe:O.” Applied Physics Letters 95 (1): 011103-7.

[6] Marti, A., Antolin, E., Linares, P. G., and Luque, A. 2012. "Understanding Experimental Characterization of Intermediate Band Solar Cells." Journal of Materials Chemistry 22 (43): 22832-9.

[7] Tanaka, T., Yu, K. M., Levander, A. X., Dubon, O. D., Reichertz, L. A., Lopez, N., Nishio, M., and Walukiewicz, W. 2011. "Demonstration of $\mathrm{ZnTe}_{1-\mathrm{x}} \mathrm{O}_{\mathrm{x}}$ Intermediate Band Solar Cell." Japanese Journal of Applied Physics 50(8R): 082304.

[8] Luque, A., Martí, A., and Stanley, C. 2012. "Understanding Intermediate-Band Solar Cells." Nature Photonics 6 (February): 146-52.

[9] Popescu, V., Bester, G., Hanna, M. C., Norman, A. G., and Zunger, A. 2008. "Theoretical and Experimental Examination of the Intermediate-Band Concept for Strain-balanced (In, Ga)As/Ga(As, P) Quantum Dot Solar Cells." Physical Review B 78(November): 205321.

[10] López, N., Reichertz, L. A., Yu, K. M., Campman, K., and Walukiewicz, W. 2011. "Engineering the Electronic Band Structure for Multiband Solar Cells." Physical Review Letters 106 (2): 028701.

[11] Ahsan, N., Miyashita, N., Islam, M. M., Yu, K. M., Walukiewicz, W., and Okada, Y. 2012. "Two-photon Excitation in an Intermediate Band Solar Cell Structure." Applied Physics Letters 102 (17): 172111.

[12] Tanaka, T., Miyabara, M., Nagao, Y., Saito, K., Guo, Q., Nishio, M., Yu, K. M., and Walukiewicz, W. 2013. "Crystal Structure and Properties of $\mathrm{Cd}_{\mathrm{x}} \mathrm{Zn}_{1-\mathrm{x}} \mathrm{O}$ Alloys across the Full Composition Range." Applied Physics Letters 102 (5): 052111.

[13] Han, D. H., Choi, J. S., and Park, S. M. 2003. "Electrochemical Preparation of Zinc Telluride Films on Gold Electrodes." Electrochemical Society 150 (45): C342-6.

[14] Skhouni, O., El Manouni, A., Mollar, M., Schrebler, R., and Marí, B. 2014. "ZnTe Thin Films Grown by Electrodeposition Technique on Fluorine Tin Oxide Substrates." Thin Solid Films 564 (August): 195-200.

[15] Ullah, H., Soucase, B. M., Skhouni, O., and El Manouni, A. 2014. A Numerical Simulation Study of ZnTe-Based Solar Cells. IEEE, ISBN 978-1-4799-7336-1.

[16] Skhouni, O., El Manouni, A., and Marí, B. 2016. "Numerical Study of the Influence of ZnTe Thickness on CdS/ZnTe Solar Cell Performance.” European Physical 
Journal Applied Physics 74 (2): 24602.

[17] Merz, J. L. 1968. "Isoelectronic Oxygen Trap in ZnTe." Physical Review 176 (3): 961-8.

[18] Cuthbert, J. D., and Thomas, D. G. 1967. "Fluorescent Decay Times of Excitons Bound to Isoelectronic Traps in GaP and ZnTe." Physical Review 154 (3): 763-71.

[19] Burgelman, M., Nollet, P., and Degrave, S. 2000. "Modelling Polycrystalline Semiconductor Solar Cells." Thin Solid Films 361-362 (February): 527-32.

[20] Burgelman, M., Verschraegen, J., Degrave, S., and Nollet, P. 2004. "Modeling Thin Film PV Devices." Progress in Photovoltaics 12 (2-3): 143-53.

[21] Verschraegen, J., and Burgelman, M. 2007. "Numerical Modeling of Intra-Band Tunneling for Heterojunction Solar Cells in Scaps." Thin Solid Films 515 (15): 6276-9.

[22] Movla, H. 2014. "Optimization of the CIGS Based Thin
Film Solar Cells: Numerical Simulation and Analysis." Optik 125 (1): 67-70.

[23] Neimegeers, A., Gillis, S., and Burgelman, M. 1965. "A User Program for Realistic Simulation of Polycristalline Heterojunction Solar Cells: SCAPS-1D.” In Proceedings of the 2nd World Conference on Photovoltaic Energy Conversion JRC, European Commission, 672-5.

[24] Lucovsky, G. 1965. "On the Photoionization of Deep Impurity Centers in Semiconductors." Solid State Commun 3(9): 299-302.

[25] Tobias, I., Luque, A., and Marti, A. 2010. "Numerical Modeling of Intermediate Band Solar Cells." Semiconductor Science and Technology 26 (1): 014031.

[26] Lin, A. S., Wang, W., and Phillips, J. D. 2009. "Model for Intermediate Band Solar Cells Incorporating Carrier Transport and Recombination." Journal of Applied Physics 105 (6): 064512. 